\title{
Using existing LMS technology and learning analytics data to support student learning
}

\author{
Xingxang Wang \\ University of Auckland \\ xingang.wang@auckland.ac.nz \\ Sally Eberhard \\ University of Auckland \\ s.eberhard@auckland.ac.nz
}

Select Submission Type: Brief Presentation (20mins + 10minsQ\&A)

Keywords: LMS, Learning Analytics, Student Learning

\section{Abstract:}

This presentation is about a teaching staff's journey of incremental use of technologies to support students' learning.

Graduate School of Management offers several master degrees through the Business Masters programme for students who do not necessarily have a business background. The programme is designed for a cohort to work through the courses together, the cohorts come from diverse backgrounds. English is a second language for most of the students. The author teaches in one of the core courses in the programme (Economics for Managers), the course has a team-based learning session where lectures are held, then students have a two-hour tutorial with the author. The students are supposed to be prepared by completing their worksheet before the tutorials. Yet students rarely do, so they come to the tutorials unprepared, unable to answer many of the questions, and often still confused over the new concepts introduced to them that week. The author wanted to use some of the techniques from the flipped classroom approach to help students prepare for the tutorial. After consulting with a learning designer, the teaching staff and the learning designer turned the paper-based tutorial worksheet into online quizzes using the quiz function in the LMS.

These quizzes are set up with $0 \%$ weighting and they close off before the tutorial starts. Students can work through the questions and get hints and additional resources on the topic for them to work through, but they don't get the answers until the tutorial time.

After the first quiz closed, author noticed a big improvement in the classroom, students were more engaged and they felt comfortable answering questions in class. The quiz statistics also allowed author to focus his energy in class on topics and areas where students were more confused and skip the topics students understood well. Student feedback to these preparation quizzes were positive.

After the success of utilizing existing technology to change how the author supports students, the author was introduced to another tool, OnTask. The author obtained student data from these online quizzes, as well as other learning analytics data from LMS (e.g., participation, page view, test results). Through further analysis, the author was able to use the OnTask tool to provide detailed and individualized feedback to the students on the topic areas that they may be weaker on and provide guidance to these students. The individualised feedback is expected to help students to improve their academic performance and attainment. Students could potentially feel more valued, as the feedback they receive is generated according to their own personal assessments and so is likely to enhance their engagement.

During the presentation, the author will share the journey of how both tools were used, data sources and analysis, as well as the results and feedback from the students, plus the teachers' reflection. These "experiments" with using technology to support student learning has changed how the author's teaching practices. The author has shared experience with other colleagues. The author's experience will also be beneficial to others who are seeking to use technology to enhance their teaching practices and to better support their students. 